\section{Abordaje diagnóstico y terapéutico de la hemorragia subaracnoidea}

Diagnostic and therapeutic approach to subarachnoid hemorrhage

Wellington Patricio Arce Chariguaman

Médico general, Hospital General Riobamba

IESS, wellingtonarcepch@hotmail.com,

https://orcid.org/0000-0001-7260-3327

Jenniffer Stefania Parra Quintana

Médico general, Clínica Kennedy Alborada,

jennifferparra91@gmail.com,

https://orcid.org/0000-0002-0213-4681

Ninika Alejandra Yuniz Molina

Médico general, Hospital General del Norte de

Guayaquil Los Ceibos,

dra.ninikayunizm@gmail.com,

https://orcid.org/0000-0002-2547-032X

Lissette Esther Villagrán

Médico general, Hospital General Guasmo Sur,

md.villagran1987@gmail.com,

https://orcid.org/0000-0002-4992-4816

Guayaquil - Ecuador
http://www.jah-journal.com/index.php/jah

Journal of American health

E-1

Esta obra está bajo una Licencia Creative Commons

Atribución-NoComercial-Compartirlgual 4.0 Internacional.

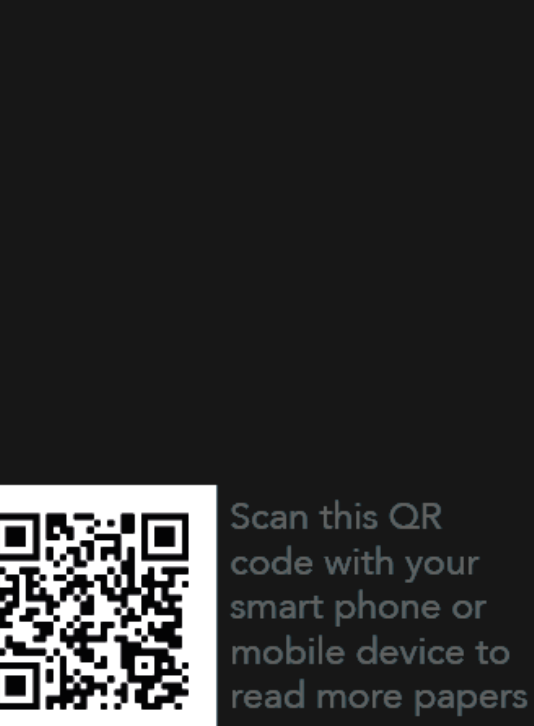

\section{RESUMEN}

La cefalea es uno de los motivos más comunes de presentación en el servicio de urgencias, la mayoría son benignas, pero es imperativo comprender y discernir las causas que amenazan la vida cuando se presentan. El dolor de cabeza causado por una hemorragia subaracnoidea por un aneurisma roto es uno de los más mortales, con una letalidad significativa. El objetivo de este estudio es actualizar los referentes teóricos de la hemorragia subaracnoidea, métodos de diagnóstico y del manejo terapéutico. Se realizó una búsqueda sistemática de investigaciones recientes en bases de datos de Elsevier, Pubmed y Scopus de articulos publicados entre el 2013 al 2020, de idioma inglés y español. La causa más frecuente de la HSA es el aneurisma cerebral y abordaje quirúrgico se fundamenta en evitar la aparición de dos de sus principales complicaciones neurológicas como el resangrado y el vasoespasmo. En los pacientes no tratados quirúrgicamente es frecuente que recidive la hemorragia. El estudio de elección es la Resonancia magnética y la angiografía, siendo el nimodipino la terapia recomendada para la prevención de la isquemia cerebral.

PALABRAS CLAVE: hemorragia subaracnoidea, terapéutica, resangrado, isquemia, aneurisma.

\begin{abstract}
Headache is one of the most common reasons for presentation in the emergency department, most are benign, but it is imperative to understand and discern the life-threatening causes when they present. Headache caused by subarachnoid
\end{abstract}


hemorrhage from a ruptured aneurysm is one of the most deadly, with significant fatality. The objective of this study is to update the theoretical references of subarachnoid hemorrhage, diagnostic methods and therapeutic management. A systematic search of recent research was carried out in Elsevier, Pubmed and Scopus databases of articles published between 2013 and 2020, in English and Spanish. The most common cause of $\mathrm{SAH}$ is brain aneurysm, and the surgical approach is based on avoiding the appearance of two of its main neurological complications, such as rebleeding and vasospasm. In patients not treated surgically, bleeding is common. The study of choice is MRI and angiography, nimodipine being the recommended therapy for the prevention of cerebral ischemia.

KEY WORDS: subarachnoid hemorrhage, therapeutic, rebleeding, ischemia, aneurysm.

\section{INTRODUCCIÓN}

La causa más frecuente de un sangrado espontáneo es la rotura de un aneurisma, que, se traduce como un sangrado súbito en el espacio subaracnoideo (La hemorragia subaracnoidea HSA). Los síntomas incluyen la cefalea súbita y grave, habitualmente con pérdida o deterioro de la conciencia. Es frecuente el vasoespasmo secundario (que produce una isquemia cerebral focal), el meningismo y la hidrocefalia. El diagnóstico se realiza mediante la TC o la RM; si los estudios de neuroimagen son normales, el diagnóstico se realiza con un análisis del líquido cefalorraquídeo y el tratamiento se enfoca a través de medidas sintomáticas y neurocirugía o medidas endovasculares, preferentemente en un centro integral para el tratamiento del accidente cerebrovascular (1).

El $80 \%$ de las HSA están originadas por la rotura de aneurismas intracraneales. Existen factores de riesgo asociados a éstos como son el consumo de alcohol (más de 40 gramos), el tabaco, la hipertensión arterial, el embarazo, el parto, los anticonceptivos orales y las sustancias tóxicas como la cocaína. Además, destaca el factor heredo familiar, como ocurre en los casos de coartación de aorta, riñones poliquísticos, enfermedad del colágeno tipo III, neurofibromatosis I y Síndrome de Marfan. (2). Como en todo paciente crítico, las primeras medidas son el $A B C$ (vía aérea, ventilación y oxigenación y estabilización cardiovascular). Las medidas generales de tratamiento, consagradas por la práctica diaria, pero de las que existe, en general, escaso nivel de evidencia. Luego se evalúa la función neurológica: se debe identificar la causa de cualquier disminución del nivel de conciencia o déficit focal antes que atribuir esos signos al efecto del evento inicial. (3).

Comúnmente se origina, debido a la rotura de una aneurisma, y casos menos frecuentes, a raíz de una malformación arteriovenosa, un traumatismo $\mathrm{u}$ otros problemas de los vasos sanguíneos o de salud pueden provocar el sangrado. No obstante, cualquiera sea su causa, al no ser tratada, puede ocasionar un daño cerebral permanente e incluso la muerte. (4).

El objetivo de esta revisión literaria es proporcionar una actualización de los conceptos más importantes de La hemorragia subaracnoidea sobre todos su abordaje diagnóstico y terapéutico. 


\section{MATERIALES Y MÉTODOS}

Se realizó una búsqueda bibliográfica en PubMed y Scielo, Scopus, Google Scholar entre otros buscadores de los últimos 10 años previos a esta publicación y otros de relevancia como precedentes de este trabajo. Se incluyeron estudios de revisión narrativa o sistemática sobre métodos diagnósticos y abordaje terapéutico actual, escritos en idioma inglés o español. Se excluyeron los artículos sobre cartas a los editor y memorias de congresos. Se proyectó un total de 40 artículos, de los cuales se seleccionaron 29 investigaciones completas. De estos, 10 artículos no eran elegibles, ya que no contenían descripción evaluación diagnóstica $y$, por lo tanto, se excluyeron. Se utilizaron descriptores de ciencias de la salud (DeCS) y los Medical Subject Headings (MeSH), combinados con marcadores interboleoanos para reducir la búsqueda e identificación de los artículos científicos relacionados al tema de estudio.

\section{RESULTADOS}

El tratamiento médico de la HSA tiene como principal objetivo situar al paciente en las mejores condiciones clínicas para que se pueda abordar la exclusión de la circulación del aneurisma roto con las máximas garantías. Así, en estos casos y en aquellos en los que no existe una etiología aneurismática, se pretende evitar la aparición de dos de sus principales complicaciones neurológicas: el resangrado y el vasoespasmo, así evitar el propio vasoespasmo si este llega a producirse. (5).

La hemorragia subaracnoidea puede producirse por un traumatismo cráneo encefálico o cuando el aneurisma se rompe produciendo flujo de sangre en el espacio subaracnoideo; Posteriormente a esta injuria inicial, se manifiesta un efecto deletéreo sobre varias zonas del cerebro que desencadena una cascada inflamatoria, además de varios fenómenos vasculares y estructurales que pueden confluir ocasionando hipoflujo-isquemia e infarto cerebral $(2,4)$.

Generalmente, la enfermedad aneurismática cerebral no se puede determinarse tempranamente; por lo que, no es hasta que se manifiesta la ruptura de la pared del vaso y se convierte en una hemorragia subaracnoidea. (6) La intensidad del sangrado subaracnoideo es el elemento más importante para el pronóstico de estos pacientes, de ahí que la mortalidad se presenta en más del 83,3\% de los enfermos que se encuentran en estados III - IV de Fisher, según referencia del trabajo factores de mal pronóstico en la evolución del paciente con diagnóstico de HSA realizada en Cuba. (7).

Debe tratarse las condiciones o factores asociados como la HTA, la discrasia sanguínea y el punto hemorrágico, mediante hemostasia instrumental $u$ obliteración neuroquirúrgica del aneurisma. En unos pocos pacientes, la confusión y la recuperación motora retardada persisten durante semanas por hidrocefalia comunicante secundaria, que requiere derivación ventricular cerebral. En los pacientes no tratados quirúrgicamente es frecuente que recidive la hemorragia. (8).

\section{Fisiopatología}

El ochenta y cinco por ciento de los casos de HSA atraumática son el resultado de la rotura de un aneurisma. Etiologías alternativas incluyen hemorragia perimesencefálica, que tiene un curso benigno, así como malformaciones arteriovenosas, fístula arteriovenosa dural, disección arterial, aneurisma micótico y abuso de cocaína. La prevalencia de 
aneurismas en la población general es aproximadamente de 2 a 5\%, mayor en aquellos con antecedentes familiares de aneurismas $\mathrm{y} / \mathrm{o}$ antecedentes personales de Ehlers-Danlos o enfermedad renal poliquística. No todos los aneurismas son peligrosos $(5,7)$. Los factores asociados con el riesgo de ruptura incluyen hipertensión, tabaquismo, consumo excesivo de alcohol, simpaticomiméticos, raza negra, etnia hispana y tamaño del aneurismático $>10$ milímetros $(\mathrm{mm})$. La HSA aneurismática es más común en mujeres y en pacientes de 40 a 60 años (8).

Los aneurismas se presentan típicamente en los puntos de bifurcación de la arteria cerebral en las regiones anterior o posterior.Se ha teorizado que la fisiopatología aneurismática implica debilidad congénita en la pared del vaso o cambios degenerativos que dan como resultado la destrucción de la elasticidad de la pared del vaso en puntos de gran turbulencia, como las bifurcaciones (8).

\section{Clasificación}

Existen varios sistemas de clasificación para la HSA. La puntuación de Hunt y Hess y el sistema de clasificación de la Federación Mundial de Cirujanos Neurológicos se utilizan para predecir el resultado del paciente, y el grado de Fisher ayuda a predecir el vasoespasmo. Dada la derivación retrospectiva de estas escalas y poca o ninguna evaluación de la variabilidad intra e interobservador, no se puede recomendar una sola escala sobre otras (4). En cuanto a los resultados y el pronóstico centrados en el paciente, no se observó que las puntuaciones específicas funcionen mejor que la Escala de coma de Glasgow (GCS) (9).
Sin embargo, los sistemas de clasificación ayudan a resaltar un concepto importante de sesgo de espectro. A medida que profundizamos en el diagnóstico de HSA, es importante tener en cuenta que algunos pacientes con HSA, por ejemplo, los pacientes de Hunt y Hess grados I y II, se pasan por alto con mayor frecuencia porque los síntomas son más leves y pueden tener aneurismas más pequeños con menos sangre subaracnoidea. Estos pacientes no necesariamente mejoran o tienen menos morbilidad con rotura o rerotura (9).

Cuadro 1. Clasificación de Hunt y Hess para la hemorragia subaracnoidea.

\begin{tabular}{|c|c|c|}
\hline Calificación & Criterios & Supervivencia \\
\hline 1 & Cefalea asintomática o leve con ligera rigidez de nuca & $70 \%$ \\
\hline II & $\begin{array}{c}\text { Dolor de cabeza de moderado a intenso, rigidez de nuca, sin } \\
\text { déficit neurológico aparte de la parálisis de los pares } \\
\text { craneales }\end{array}$ & $60 \%$ \\
\hline III & Somnolencia, confusión o déficit focal leve & $50 \%$ \\
\hline IV & $\begin{array}{l}\text { Estupor, hemiparesia de moderada a grave, posiblemente } \\
\text { rigidez de descerebración temprana o alteración vegetativa }\end{array}$ & $20 \%$ \\
\hline V & $\begin{array}{c}\text { Coma profundo, rigidez descerebrada, apariencia } \\
\text { moribunda }\end{array}$ & $10 \%$ \\
\hline
\end{tabular}

Métodos diagnósticos

La valoración inicial neurológica comienza con la secuencia clásica ABC (vía aérea, ventilación $y$ oxigenación $y$ estabilidad cardiovascular), en caso de observar un bajo nivel de conciencia en el paciente se plantea la intubación orotraqueal (IOT) y ventilación mecánica en pro de cuidar que la saturación de oxígeno (SatO2) se encuentre por encima del 95\% y una presión parcial de dióxido de carbono (pCO2) normal, evitando en cuanto sea posible la hiperventilación. (9).

La tomografía computarizada (TC) craneal sin contraste es el primer escalón diagnóstico de la HSA. Cuando la TC se realiza en las primeras 24 horas del inicio de los síntomas se puede observar en el 92\% de los casos la hiperdensidad de señal de la sangre en el espacio subaracnoideo. La 
sensibilidad diagnóstica de la TC decrece a medida que se va reabsorbiendo la sangre del espacio subaracnoideo, por lo que, ante la sospecha clínica y si la TC es negativa, se ha de realizar una punción lumbar (PL). Si ésta fuera negativa, la sintomatología tuviera menos de 12 horas de evolución y persistiera una gran sospecha de HSA, determinadas secuencias de resonancia magnética (RM) pueden resultar diagnósticas. (10).

Con respecto a la Angiografía cerebral, se obtendrán imágenes más detalladas se realizará si se sospecha la presencia de una hemorragia subaracnoidea, sobre todo si la causa no está clara o no aparece en otras imágenes. (11).

Las complicaciones precoces más graves de esta patología son la ocurrencia de una nueva hemorragia o la obstrucción aguda de las vías de circulación del líquido cerebroespinal (LCE) produciendo una hidrocefalia aguda. Más tardíamente pueden aparecer vasoespasmo o alteraciones crónicas de reabsorción del LCE. El riesgo de vasoespasmo (y eventuales infartos secundarios) y de hidrocefalia están directamente relacionados a la magnitud de la hemorragia inicial. (12).

\section{Tratamiento}

El tratamiento inicial con analgésico señala la pauta inicial se realizará con Paracetamol 1gr/8h/ y/o Dexketoprofeno $50 \mathrm{mg} / 8 \mathrm{~h} /$ (no prolongando este último más de 3 días por el riesgo de insuficiencia renal), y en los casos de cefalea refractaria valorar el uso de tramadol $100 \mathrm{mg} / 8 \mathrm{~h} /$ o Meperidina $1 \mathrm{mg} / \mathrm{kg} / 8 \mathrm{~h} / \mathrm{sc}$, asociando profilaxis antiemética con Metoclopramida 1 amp/8h/ev. Por otro lado, la Dexametazona en bolus de $8 \mathrm{mg} / \mathrm{ev}$ se utilizará en caso de cefalea persistente secundaria a síndrome meníngeo. (13).
El único fármaco que ha mostrado reducción de eventos adversos y ha mejorado el pronóstico de los pacientes con HSA es la nimodipina vía oral en dosis de $60 \mathrm{mg}$ cada 4 horas por 21 días, siempre y cuando se inicie dentro de los primeros 4 días de ocurrido el evento y se inicie antes de la presencia de VEC. Si bien, esta evidencia adolece del número de pacientes necesario para se evidencia tipo 1 , el tiempo ha ratificado su uso cuando es iniciada en los primeros 4 días de la HSA. (14)

Desde varios años se ha estudiado que los pacientes que recibían $1 \mathrm{~g}$ intravenoso de ácido tranexámico en el momento del diagnóstico de la HSA, seguido de $1 \mathrm{~g} / 6 \mathrm{~h}$ hasta la oclusión del aneurisma, asociaban significativamente menores tasas de resangrado y mejor evolución clínica. En esta línea de actuación, ha observado que disminuye las cifras de resangrado sin que aparezcan complicaciones isquémicas mayores. Se observó una mayor tasa de trombosis venosa profunda, pero no de embolia pulmonar. La mortalidad fue similar con respecto al grupo placebo, pero el pronóstico mostró tendencia no significativa a la mejoría en el grupo tratado. (5)

El uso de embolización con espiral, para sellar el vaso sanguíneo, no requiere una craneotomía (15). Para el tratamiento endovascular se emboliza la lesión por medio de depósitos de espirales de platino (coils) colocados en el seno del aneurisma por medio de un catéter localizado en la arteria femoral. Este procedimiento está indicado en pacientes (>70 años), en HSA graves (WFNS $\mathrm{VI}$ y $\mathrm{V}$ ) y aneurismas de arteria basilar (16).

La colocación de stents se debe considerar solo si otras técnicas menos agresivas han sido descartadas se prefiere 
tratamiento endovascular debido a la baja probabilidad de complicaciones posterior al procedimiento y siempre se debe realizar un control del aneurisma posterior al procedimiento para valorar la oclusión completa del aneurisma. (16).

\section{DISCUSIÓN}

Es vital que una hemorragia cerebral sea tratada inmediatamente, de esta manera se podrá salvar la vida del paciente y se reducirá el riesgo de invalidez. La técnica para emplear dependerá del tipo y de la ubicación de la hemorragia, pero en general se focalizará en restaurar el flujo sanguíneo y en reducir la presión en el cerebro. El coágulo puede tratarse con fármacos que deben administrarse en las primeras horas; Otras técnicas serían la cirugía y los tratamientos vasculares intracraneales. (17) La Colocación de un clip en el aneurisma a través de una craneotomía para acceder al cerebro, con la finalidad de prevenir que vuelva a sangrar.

Cuando existe una sospecha clínica de HSA basada en la historia y el examen físico, la tomografía computarizada (TC) sin contraste es la primera herramienta de diagnóstico. También es valioso para excluir otras patologías como hemorragia intracraneal, neoplasias malignas o abscesos. Al inicio del sangrado, la sangre subaracnoidea es la más fácilmente visible en la TC, pero se vuelve más difícil de apreciar a medida que avanza la degradación de los glóbulos rojos (RBC) (15). Los avances en neuroimagen han aumentado la sensibilidad de la TC sin contraste, lo que plantea dudas sobre la necesidad de realizar una punción lumbar $(\mathrm{PL})$ ante una TC negativa.

Un metanálisis publicado en 2016 intentó responder la pregunta de la sensibilidad de la TC con relación al tiempo desde el inicio de los síntomas. El análisis, que incluyó cinco estudios, evaluó a pacientes con cefalea en trueno y examen neurológico normal. Si bien los resultados tienen muchas de las limitaciones de un metanálisis, un análisis estadístico conservador mostró que una TC sin contraste completada dentro de las seis horas posteriores al inicio del dolor de cabeza tenía una sensibilidad del $98,7 \%$ con intervalos de confianza del $97,1 \%$ al $99,4 \%$ (16). Los autores tomaron en consideración los siguientes criterios: el paciente debe tener un hematocrito $>30 \%$ y una cefalea en trueno aislada sin convulsiones, síncope o dolor de cuello; y la imagen de TC debe ser de tercera generación o más reciente, de alta calidad, leída por un radiólogo de nivel asistente y evaluada con la indicación de imagen como dolor de cabeza en trueno o preocupación por HSA.Si se cumplen estos criterios,

Si la TC de cabeza sin contraste no es definitiva la siguiente herramienta de diagnóstico recomendada es la punción lumbar (PL). En estos casos, el LP está buscando dos elementos que susciten preocupación por la HSA: 1) glóbulos rojos; y 2) xantocromía (bilirrubina en el líquido cefalorraquídeo [LCR]). Dada la sensibilidad del TC discutida anteriormente, la toma de decisiones compartida debe llevarse a cabo con respecto al PL $(15,16)$. En particular, con una sensibilidad cercana al 99\% para un estudio adecuado si se completa dentro de las seis horas y cumple con los criterios descritos anteriormente.

En este contexto, los riesgos (eventos adversos y falsos positivos) generalmente superan a los beneficios y se desaconseja la LP. Hay casos raros en los que el escenario 
clínico sugiere una HSA tan fuertemente que incluso una TC negativa adecuada completada dentro de las seis horas no puede descartar HSA y debe ser seguida por LP. Si las imágenes se completan después del período de tiempo de seis horas, la sensibilidad de la TC cae al $85,7 \%$. En estos casos, la utilidad diagnóstica de la LP aumenta a medida que aumenta la probabilidad de HSA después de una TC negativa. En tales casos, se indica LP (15). También debe tenerse en cuenta que, en consonancia con la baja prevalencia de esta enfermedad, un estudio reciente mostró que aproximadamente el 0,4\% de los PL revelaron aneurismas. Todavía se recomienda la toma de decisiones compartida, como con cualquier procedimiento invasivo (17).

Los glóbulos rojos intactos se observarán al comienzo de la enfermedad y disminuirán a medida que las células se descomponen y se reabsorben. De acuerdo con la fisiopatología, se cree que la presencia de glóbulos rojos en el cuarto tubo de LCR representa HSA. Desafortunadamente, la PL es a menudo un procedimiento técnicamente difícil y las tasas de "punción traumática" o introducción de eritrocitos por trauma local y manipulación de la aguja pueden acercarse al 30\%. Esto complica el diagnóstico de HSA por los resultados de los glóbulos rojos (18). Debido a que diferenciar entre una HSA verdadera y una punción traumática es de suma importancia clínica, los autores han investigado criterios para ayudar a diferenciar los dos.

La verdadera xantocromía es patognomónica de la HSA. Esto es valioso cuando existe una alta sospecha clínica y el recuento de glóbulos rojos no está lo suficientemente elevado como para diferenciarlo de un diagnóstico de punción traumática. La xantocromía se detecta mediante inspección visual del tubo de LCR frente a un tubo de agua o mediante espectrofotometría. Los glóbulos rojos que se han vertido en el LCR a partir de la HSA finalmente se descomponen y liberan oxihemoglobina, que luego se convierte en bilirrubina in vivo, interpretada como xantocromía o literalmente "color amarillo" (18). Cabe señalar que la sangre de un grifo traumático puede producir oxihemoglobina cuando se expone a la luz natural, que puede producir un color amarillo, pero al estar fuera del cuerpo no producirá bilirrubina. Proteger la muestra de la luz minimizará la conversión de glóbulos rojos en oxihemoglobina.

Durante la última década, la angiografía por tomografía computarizada (ATC) del cerebro se ha convertido en parte de la discusión para descartar la HSA. Como medio no invasivo para resaltar la anatomía vascular y detectar aneurismas, la ATC tiene muchas ventajas. Al igual que la TC de cerebro sin contraste, los avances en neuroimagen han demostrado que la ATC tiene una sensibilidad de hasta el $98 \%$ y una especificidad del $100 \%$ para los aneurismas en pacientes con HSA conocida. Estas estadísticas se derivan de un pequeño conjunto de datos $(n=65)$ donde los resultados de la ATC se compararon con los estándares de oro de la angiografía por sustracción digital o los hallazgos quirúrgicos (19).

Según la mejor bibliografía disponible, una ATC sin hallazgos de aneurisma cuando se combina con una TC de cabeza sin contraste negativa tiene una probabilidad de enfermedad posterior a la prueba de < $1 \%$. Este porcentaje es importante porque cae por debajo del umbral de prueba de 
la mayoría de los médicos, que es la probabilidad de enfermedad por debajo de la cual no se requiere más investigación. La sensibilidad de la ATC es del 92,3\% para los aneurismas $<4 \mathrm{~mm} \mathrm{y}$, a diferencia de las patologías en las que el tamaño de la lesión se correlaciona con la gravedad de la enfermedad (embolia pulmonar), un aneurisma cerebral pequeño roto todavía puede provocar una morbilidad significativa y mortalidad (20).

En los casos de HSAa de bajo grado (WFNS 1, 2 y 3), el tratamiento del aneurisma debe realizarse antes de $24 \mathrm{~h} 1$ a partir del momento en que se confirma el diagnóstico de HSAa. En los casos de HSAa de alto grado (WFNS 4 y 5), el mejor timing para el tratamiento del aneurisma debe ser discutido caso a caso por un equipo compuesto por neurocirujano, neurorradiólogo intervencional e intensivista. En los casos en que se decida diferir el tratamiento del aneurisma, se debe considerar el tratamiento con ácido tranexámico 1 gr cada $6 \mathrm{~h}$ hasta la exclusión aneurismática o, máximo, por 72 h. (18).

La terapia más utilizada para evitar el déficit isquémico es la "terapia Triple $\mathrm{H}^{\text {" }}$ (hemodilución hipervolémica hipertensiva), para que así a través de los vasos con luz disminuida, la sangre pueda transitar con mayor facilidad y llegar finalmente al tejido isquémico. (19). Es controvertido aun el momento para realizar la cirugía, se recomienda el clipado quirúrgico para reducir en forma drástica la posibilidad de resangrado, la cirugía precoz (3 días) tiene la ventaja de prevenir el resangrado $y$ posibilitar el tratamiento adecuado del vasoespasmo. Todos aquellos casos en los que no se cuente con todos los recursos humanos y tecnológicos adecuados. (20).

Los bloqueantes cálcicos son drogas extensamente estudiadas para la prevención del déficit isquémico tardío debido a vasoespasmo, siendo la nimodipina el exponente de este grupo más ampliamente estudiado en pacientes con HSA aneurismática, han demostrado una disminución en forma estadísticamente significativa del déficit neurológico severo debido a vasoespasmo cerebral a los 21 días. En otro estudio, también de nivel II, con una muestra más pequeña, también de pacientes en buen grado, la nimodipina por vía oral demostró reducir la ocurrencia de déficit neurológico debido a vasoespasmo. (21). De este grupo de fármacos la nimodipina es el que mejores resultados ha demostrado. La nimodipina es un antagonista de los canales de calcio dependientes de voltaje tipo-L. El efecto benéfico de la nimodipina se ha demostrado en diferentes series con reducción del riesgo absoluto de $5.1 \%$ y número necesario a tratar de 20 enfermos, con efectos neuroprotectores más que efectos en la vasculatura cerebral, y sin efecto en la mortalidad con respecto a placebo. (22)

El tratamiento de un aneurisma roto es multifactorial y por lo tanto multidisciplinario. Implica, por una parte, eliminar precozmente la causa de la hemorragia y por otro lado anticipar, prevenir y corregir fenómenos asociados a la presencia de sangre en el espacio subaracnoideo, principalmente vasoespasmo y/o hidrocefalia. La mantención del equilibrio del medio interno, de la hemodinamia y de la función pulmonar juegan un rol crucial en el desenlace. El detalle del manejo por parte del intensivista en estos pacientes está fuera de los objetivos de este trabajo. (23)

Otros factores relevantes son el tratamiento de la hiperglucemia aparece en $1 / 3$ de los pacientes con HSA. Es importante 
el control glucémico ya que, glucemias > $180 \mathrm{mg} / \mathrm{dl}$ se asocian con una peor situación clínica al ingreso y mal pronóstico a medio plazo. Se recomienda insulina rápida sc a partir de glucemias mayores a $150 \mathrm{mg} / \mathrm{dl}$. La situación ideal sería el mantenimiento de las glucemias entre 120$150 \mathrm{mg} / \mathrm{dl}$. (24).

Otras técnicas usadas desde el 2005, Castillo y col., evaluaron el tratamiento de los aneurismas cerebrales a través de la técnica endovascular, fue un procedimiento seguro y efectivo al aplicarlos en aneurismas pequeños $y$ medianos. La mortalidad fue significativamente menor en comparación con el grupo de clipaje quirúrgico de pacientes en similares condiciones clínicas. (25)

Otros ejercicios empleados indistintamente por las fases de rehabilitación que ha pasado la paciente como los Ejercicios de distensión que se emplean cuando necesitamos incidir en los tejidos patológicamente alterados con el objetivo de aumentar sus propiedades Elásticas. Este ejercicio tienes una importancia fundamental en el tratamiento de contracturas, parálisis y dificultades articulares. (26).

Es claro que la cirugía temprana puede conducir a edema, hemorragia posquirúrgica hasta hallar dificultad para ubicar al aneurisma durante el acto quirúrgico. Entre las ventajas de la cirugía tardía están un cerebro menos edematoso y menos inestabilidad cerebrovascular durante la cirugía. (27).

Pese a que la presencia de convulsiones no es inusual tras la ocurrencia de una HSA aún sigue siendo motivo de debate la idoneidad del uso de profilaxis anticonvulsivante en estos pacientes. Panczykowski D. y col. evaluaron la incidencia de en un estudio retrospectivo en el que se usaron anticonvulsivantes de forma rutinaria, y concluye que una profilaxis anticonvulsiva en el contexto de un paciente con HSA no está indicada de manera rutinaria [22]. (28) (29).

\section{CONCLUSIONES}

La hemorragia subaracnoidea es una morbilidad relativamente compleja y grave y su área terapéutica debe ser tratada en hospitales de alta complejidad con métodos y terapéutica oportuna y adecuada, sobre todo en los casos de personas jóvenes sanos. A pesar de los avances en el diagnóstico y tratamiento de la hemorragia subaracnoidea aneurismática, la mortalidad sigue siendo alta. El tratamiento temprano se basa en la reparación final del aneurisma a través de dos enfoques posibles: pinzamiento microvascular neuroquirúrgico o enrollamiento mediante acceso endovascular.

\section{REFERENCIAS}

1. Chong JY. MANUAL MSD Versión para profesionales. [Online].; 2020. Available from: https://www.msdmanuals.com/esec/professional/trastornosneurol\%C3\%B3gicos/accidentecerebrovascular/hemorragia-subaracnoideahsa.

2. Formento $A E$, Álvarez RF, Ros Tristpán $C M$, Carpe Gil MJ, Martínez Oviedo A. Scielo. [Online].; 2008. Available from: http://scielo.isciii.es/scielo.php?script=sci_a rttext\&pid=S0212-71992008000700008.

3. Guerrero López F, de la Linde Valverde CM, Pino Sánchez FI. Manejo general en Cuidados Intensivos del paciente con hemorragia subaracnoidea espontánea. Medicina Intensiva Vol. 32. Núm. 7. 2008;: p. 342 - 353. 
4. Flores Poveda KA, Quiñonez García KJ, Flores Subia DL, Cárdenas Choez CA. Actuación clínica en la hemorragia subaracnoidea. RECIMUNDO. 2020;: p. 256 - 267.

5. Vivancos J, Gilo F, Frutos R, Maestre J, GarcíaPastor A, Quintana F, et al. Elsever. [Online].; 2014. Available from: https://www.elsevier.es/es-revistaneurologia-295-articulo-guia-actuacionclinica-hemorragia-subaracnoidea-S0213485312002496.

6. Maldonado Coronel FV, Muñoz Flores TE, Muñoz Lalangui CF, Salazar Calderón LK, Vaca Riofrío RI. Hemorragia subaracnoidea aneurismática en la unidad de cuidados intensivos del Hospi-tal Luis Vernaza, Guayaquil, Ecuador. REE Volumen 13. 2019;: p. 19 - 27.

7. Criollo P. J, Mora B. H. Tratamiento endovascular e indicadores predictivos de complicaciones y mortalidad en la hemorragia subaracnoidea espontanea de origen aneurismática. [Online]. Available from: https://www.inspilip.gob.ec/wpcontent/uploads/2017/08/15-

TRATAMIENTO-ENDOVASCULAR-E-

INDICADORES-PREDICTIVOS-DE-

COMPLICACIONES-Y-MORTALIDAD-EN-LAHEMORRAGIA-SUBARACNOIDEA-

ESPONTANEA-DE-ORIGEN-

ANEURISM\%C3\%81TIC.pdf.

8. Fundación Mapfre. Fundación Mapfre. [Online].

9. Flores Poveda KA, Quiñonez García KJ, Flores Subia DL, Cárdenas Choez CA. Actuación clínica en la hemorragia subaracnoidea. RECIAMUC 4. 2020;: p. 256 - 267.

10 Valverde Naranjo M. HEMORRAGIA SUBARACNOIDEA. REVISTA MEDICA DE COSTA RICA Y CENTROAMERICA LXXIII. 2016;: p. 221- 226.

11 MayoClinic. MayoClinic. [Online]. Available from: https://www.mayoclinic.org/eses/diseases-conditions/subarachnoidhemorrhage/diagnosis-treatment/drc20361014\#: :text=Para\%20diagnosticar\%20 una\%20hemorragia\%20subaracnoidea,detec tar\%20sangrado\%20en\%20el\%20cerebro.

12 Yáñez Lermanda A, Ruiz-Aburto Aguilar A. Tratamiento de la hemorragia subaracnoidea aneurismática en el Hospital Clínico de la Universidad de Chile. Rev. méd. Chile vol.142. 2014.

13 Lagares A, Gómez PA, Alén JF, Arikan F, Sarabia R, Horcajadas A, et al. Hemorragia subaracnoidea aneurismática: guía de tratamiento del Grupo de Patología Vascular. Scielo Neurocirugía 22. 2011;: p. 93 - 115.

14 Murillo-Bonilla LM, Magaña-Solano G, Uribe-

- Gonzalez P. Tratamiento del Vasoespasmo Cerebral en el Paciente con Hemorragia Subaracnoidea Aneurismática. Revista de Medicina Clínica Vol. 2. 2018;: p. 8 - 12.

15 Intermountain Healthcare. Intermountain Healthcare. [Online].; 2017. Available from: https://intermountainhealthcare.org/ckrext/Dcmnt?ncid=520841098.

16 Brenes Zumbado MJ, Romero Castillo A, - Jiménez Víquez M. Abordaje de hemorragia subaracnoidea. Revista Médica SinergiaVol.5 (10). 2020.

17 Topdoctors. Topdoctors. [Online]. Available

. from: https://www.topdoctors.es/diccionariomedico/hemorragia-cerebral.

18 Huidobro Salazar JF, Quintana Marín L. Guía clínica para el manejo de la hemorragia. Revista Chilena de Neurocirugía 43. 2017;: p. 156 - 165.

19 Hemorragia subaracnoidea aneurismática - con mal grado clínico: Revisión clínica. Revista Mexicana de Neurociencia 17(1). 2016;: p. 1 - 113.

20 Papalini F, Torino R, Konsol O, Bochiardo E. Recomendaciones para el Manejo de la Hemorragia Subaracnoidea Aneurismática. Revista Argentina de Neurocirugía 16: 33. 2002.

21 Dorfman BS, Previgliano IJ. RECOMENDACIONES PARA EL TRATAMIENTO MEDICO DE LA HEMORRAGIA SUBARACNOIDEA DE CAUSA 
ANEURISMATICA. MEDICINA (Buenos Aires) 60. 2000;: p. 370 - 374.

22 Carrillo Esper R, Leal Gaxiola P, Arellano

- Cervantes R. Hemorragia subaracnoidea secundaria a ruptura de aneurisma. Medigraphic Artemisa Vol. 16, núm. 1. 2009.

23 Tratamiento de la hemorragia subaracnoidea - aneurismática en el Hospital Clínico de la Universidad de Chile. Rev Med Chile 142. 2014;: p. 982 - 988.

24 SERVICIO DE URGENCIAS GENERALES

. DEPARTAMENTO DE SALUD DE SAGUNTO. PROTOCOLO DE MANEJO Y DERIVACIÓN DE LA HEMORRAGIA SUBARACNOIDEA NO TRAUMÁTICA VERSIÓN 03. Sagunto:; 2017.

25 Velásquez Salazar R, Peña EP, Puetes ED. - Terapia endovascular y hemorragia subaracnoidea. Experiencia en el Hospital Central de Maracay. Med Interna (Caracas) 29(1). 2013;: p. 52 - 61.

26 Castillo González AJ, Alba Martínez D, Alba - Martínez S, Suárez Castillo Y. Rehabilitación en paciente con una hemorragia subaracnoidea, Barrio Adentro, Zona 2, José Félix R. Venezuela. EFDeportes.com, Revista Digital № 175. 2012.

27 Hoyos Castillo JD, Moscote Salazar LR. - Hemorragia subaracnoidea aneurismática con. Revista Mexicana de Neurociencia Volumen 17. 2016;: p. 1 - 113.

28 Sabogal Barrios R, Cabrera Gonzáles A, De - Lima Zea A, Lambertinez Alvarez I, Pérez Calvo C, Pájaro Galvis N, et al. Hemorragia Subaracnoidea Aneurismática. iMedPub Journals Vol. 16 No. 6. 2017.

29 Ingelmo Ingelmo I, Rama Maceiras $P$, Hernández Palazón J, Fàbregas Julià N. RECOMENDACIONES-GUÍA DE PRÁCTICA CLÍNICA EN LA HEMORRAGIA SUBARACNOIDEA POR ROTURA DE ANEURISMA INTRACRANEAL. Revista Española de Anestesiología y Reanimacion VOLUMEN 57-SUPLEMENTO 2. 2010. 